\title{
Manejo quirúrgico de perforaciones duodenales tras colangiopancreatografía retrógrada endoscópica: serie de casos
}

\author{
Surgical management of duodenal perforation after endoscopic retrograde \\ cholangiopancreatography: case series
}

\begin{abstract}
Luis F. Cabrera-Vargas ${ }^{1,2,3,4}$, Ricardo Villarreal-Viana ${ }^{1,2,3,4,5}$, Jean A. Pulido-Segura ${ }^{3,4,6}$, Mauricio Pedraza-Ciro ${ }^{3,4 *}$, Marcia Santafe-Guerrero ${ }^{3,4}$, Laura Cabrera-Silva ${ }^{3,4}$ y David Acevedo-Fontalvo $0^{3,4}$ ${ }^{1}$ Departamento de Cirugía General, Los Cobos Medical Center, Universidad El Bosque; ${ }^{2}$ Departamento de Cirugía General, Fundación Santa Fe de Bogotá, Universidad El Bosque; ${ }^{3}$ Departamento de Cirugía General, Universidad El Bosque; ${ }^{4}$ Departamento de Medicina, Universidad El Bosque; ${ }^{5}$ Departamento de Gastroenterología, Los Cobos Medical Center, Universidad El Bosque; $6 / l$ ustrador médico. Bogotá, Colombia
\end{abstract}

\begin{abstract}
Resumen
Antecedentes: Las complicaciones tras la colangiopancreatografía retrógrada endoscópica (CPRE) que necesitan manejo quirúrgico son limitadas y aproximadamente el 0.09-1.8\% corresponden a perforación duodenal. Este tipo de lesiones requieren un diagnóstico temprano y un manejo adecuado, ya que los desenlaces pueden ser catastróficos, con una mortalidad reportada de hasta el 20\%. Objetivo: Presentar nuestra experiencia en el manejo quirúrgico de estas lesiones. Método: Estudio observacional, retrospectivo y descriptivo, de pacientes que requirieron manejo quirúrgico por perforación duodenal tras CPRE en tres centros de Bogotá, Colombia, entre enero de 2013 y diciembre de 2018. Resultados: Fueron llevados a manejo quirúrgico 13 pacientes, 7 (53.8\%) por vía laparoscópica, con una estancia hospitalaria promedio de 10.7 días y una mortalidad del 0\%, y 6 (46.1\%) por vía abierta, con una estancia hospitalaria promedio de 33 días y una mortalidad del 50\%, con seguimiento mínimo a 6 meses. Conclusiones: La perforación duodenal tras una CPRE, pese a su baja incidencia, se debe sospechar por su alta morbimortalidad. La decisión del abordaje quirúrgico está condicionada por el estado clínico del paciente, y por ende está ligada al pronóstico de este.
\end{abstract}

Palabras clave: Laparoscopia. Perforación intestinal. Complicaciones. Colangiopancreatografía retrógrada endoscópica. Cirugía.

\begin{abstract}
Background: Complications after endoscopic retrograde cholangiopancreatography (ERCP) are rare, approximately $0.09 \%$ to $1.8 \%$ are duodenal perforation. This type of injury requires early diagnosis and proper management since the outcomes can be catastrophic, leading to death in $20 \%$ of cases. Objective: To show our experience in the management of these injuries in order to establish if there is benefit from minimally invasive management versus conventional management. Method: Observational, retrospective and descriptive study comparing patients who required surgical management after post-ERCP duodenal
\end{abstract}

\footnotetext{
Correspondencia:

*Mauricio Pedraza-Ciro

Carrera 72, 81-55

Fecha de recepción: 24-07-2020

C.P. 111166, Bogotá, D.C., Colombia Fecha de aceptación: 16-10-2020

E-mail: mpedraza93@gmail.com

DOI: $10.24875 /$ CIRU.20000819

Cir Cir. 2021;89(5):611-617

Contents available at PubMed

www.cirugiaycirujanos.com

0009-7411/@ 2020 Academia Mexicana de Cirugía. Publicado por Permanyer. Este es un artículo open access bajo la licencia CC BY-NC-ND (http://creativecommons.org/licenses/by-nc-nd/4.0/).
} 
perforation in three centers in Bogotá, Colombia, between January 2013 and December 2018. Results: 13 patients were taken to surgical management after duodenal perforation post ERCP, 7 laparoscopically (53.8\%), with an average hospital stay of 10.7 days and mortality of $0 \%$ and 6 by open route (46.1\%), with an average hospital stay of 33 days and mortality of $50 \%$, with a minimum follow-up of 6 months. Conclusions: Post ERCP duodenal perforation, despite its low incidence, should be suspected due to its high mortality. Laparoscopic surgery is a good tool to address duodenal perforations after ERCP, because it presents a lower rate of complications and mortality.

Key words: Laparoscopy. Intestinal perforation. Complications. Endoscopic retrograde cholangiopancreatography. Surgery.

\section{Introducción}

Desde 1968, la colangiopancreatografía retrógrada endoscópica (CPRE) se ha usado para el tratamiento de la patología de las vías biliar y pancreática'. Solo el $10 \%$ de estos procedimientos presentan complicaciones, entre las que se reportan las perforaciones duodenales (PD) en un $0.09-1.8 \%$ de los casos $^{2,3}$.

Las PD pos-CPRE requieren un diagnóstico temprano y un tratamiento adecuado según el tipo de lesión, ya que el manejo tardío conlleva peores resultados clínicos con complicaciones como sepsis, falla multiorgánica y muerte en el $20 \%$ de los casos. El examen imagenológico de preferencia es la tomografía computarizada (TC) de abdomen, con una especificidad de hasta el $90 \%$, ya que permite observar la extravasación del contraste ${ }^{4,5}$.

La gravedad y la clínica de esta patología determinan la necesidad y el abordaje quirúrgico a realizar, pero al ser de tan baja frecuencia consideramos importante mostrar nuestra experiencia en el manejo quirúrgico abierto y laparoscópico de las PD posCPRE en nuestras instituciones.

\section{Método}

\section{Análisis estadístico}

Se realizó un estudio observacional, retrospectivo y descriptivo de pacientes con PD pos-CPRE realizada por coledocolitiasis entre enero de 2013 y diciembre de 2018 en Bogotá, Colombia, en tres instituciones: Clínica El Bosque, Policlínico del Olaya y Cardiovascular de Soacha. Los criterios de inclusión fueron presentar perforación duodenal pos-CPRE por coledocolitiasis con diagnóstico intraoperatorio y posoperatorio. Los pacientes diagnosticados con lesión duodenal posterior al procedimiento se dividieron en dos grupos: manejo médico y manejo quirúrgico por laparoscopia o abierto.

Se recopilaron datos relevantes de los registros médicos. La información revisada incluyó las siguientes variables: edad, sexo, tiempo de diagnóstico, puntuación de la American Society of Anesthesiology (ASA), diámetro de la vía biliar, comorbilidad, presencia de cálculo difícil, tipo de PD, indicación de la CPRE, presencia de pancreatitis aguda pos-CPRE, hallazgos en la TC abdominal contrastada, abordaje quirúrgico, estancia hospitalaria y mortalidad.

Los pacientes fueron valorados por un grupo multidisciplinario de gastroenterólogos, cirujanos e internistas, según su comorbilidad. Todos los pacientes tuvieron un estudio de estratificación para la coledocolitiasis con colangiorresonancia o ultrasonografía endoscópica, o ambas, previamente a la CPRE que confirmara la coledocolitiasis.

La sospecha de PD pos-CPRE se basó en los hallazgos clínicos, como signos de irritación peritoneal, paraclínicos e imagenológicos. Todos los pacientes fueron sometidos a TC contrastada abdominopélvica para evaluar el tipo de lesión. Según el tipo de PD de la clasificación de Stapfer, et al. ${ }^{6}$ y la evolución clínica del paciente, se definió si este era candidato a manejo médico o quirúrgico.

El manejo médico se basó en reposo intestinal, colocación de sonda nasogástrica, líquidos intravenosos, antibioticoterapia y control estricto de los signos vitales. Se consideró el manejo quirúrgico en los pacientes que no respondieron al manejo médico en las siguientes 12 horas, presentaron respuesta inflamatoria sistémica persistente o mostraron signos de irritación peritoneal ${ }^{7}$.

La cirugía abierta consistió en el cierre primario más exclusión pilórica con anastomosis gastroyeyunal, y la laparoscópica en cierre primario más drenajes o lavado en caso de no encontrar el sitio de la lesión. El manejo quirúrgico por cirugía laparoscópica o abierta varió según la estabilidad hemodinámica del paciente, si estaba en condiciones de soportar el neumoperitoneo, según la distensión de las asas intestinales y la disponibilidad del equipo de laparoscopia. Se realizó seguimiento durante al menos 6 meses después de la cirugía (Fig. 1). 

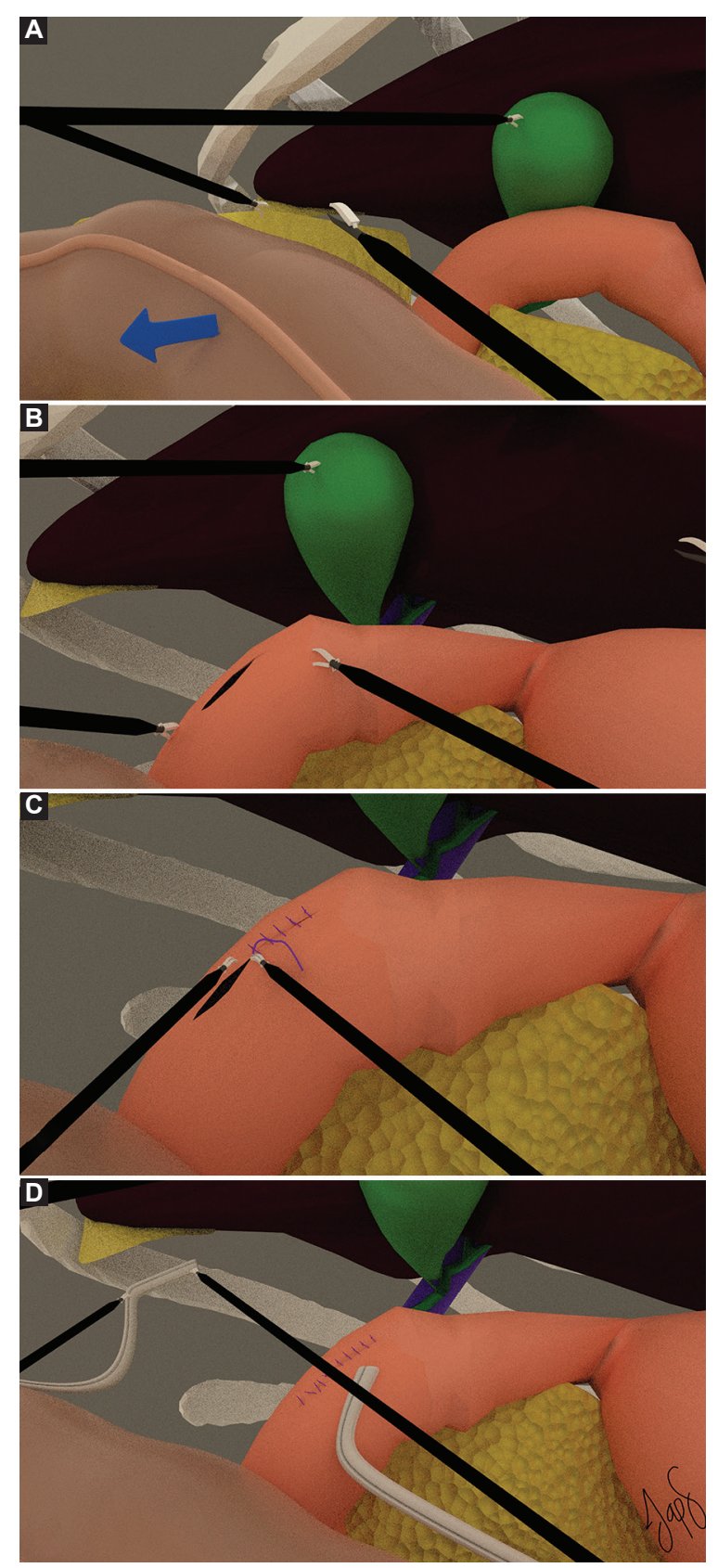

Figura 1. A: liberación del ángulo hepático del colon. B: maniobra de Kocher laparoscópica y detección de perforación duodenal. C: cierre primario de la perforación duodenal con sutura intracorpórea laparoscópica. D: drenaje del retroperitoneo con colocación de drenaje anterior y posterior a la segunda porción del duodeno.

\section{Consideraciones éticas}

Los pacientes firmaron el consentimiento informado tanto para la realización de la CPRE como para el procedimiento quirúrgico (abierto o laparoscópico). Los autores declaran que este artículo no ofrece información personal que permita identificar a los
Tabla 1. Variables demográficas

\begin{tabular}{lccc}
\hline & $\begin{array}{c}\text { Pacientes } \\
(\mathbf{n}=\mathbf{2 8})\end{array}$ & $\begin{array}{c}\text { Cirugía } \\
\text { abierta } \\
(\mathbf{n}=6)\end{array}$ & $\begin{array}{c}\text { Cirugía } \\
\text { laparoscópica } \\
(\mathbf{n}=\mathbf{7})\end{array}$ \\
\hline Mujeres & $21(75 \%)$ & $5(83.3 \%)$ & $5(71.4 \%)$ \\
Hombres & $7(25 \%)$ & $1(16.7 \%)$ & $2(28.6 \%)$ \\
Edad & 45.4 años & 37,6 años & 45 años \\
Comorbilidad & & & \\
HTA & $9(32.1 \%)$ & $1(16.7 \%)$ & $3(42.8 \%)$ \\
DM2 & $8(28.5 \%)$ & $2(33.3 \%)$ & $2(28.5 \%)$ \\
EPOC & $2(7.1 \%)$ & $0(0.0 \%)$ & $1(14.3 \%)$ \\
ASA & & & \\
I & $14(50.0 \%)$ & $3(50.0 \%)$ & $3(42.8 \%)$ \\
II & $10(35.7 \%)$ & $3(50.0 \%)$ & $2(28.5 \%)$ \\
Tiempo al diagnóstico & $4(14.3 \%)$ & $0(0.0 \%)$ & $2(28.5 \%)$ \\
Presencia de cálculo & 1.2 días & 2,3 días & 1.1 días \\
difícil & $6(21.4 \%)$ & $0(0.0 \%)$ & $4(57.1 \%)$ \\
Diámetro vía biliar & & & \\
\hline
\end{tabular}

ASA: American Society of Anesthesiology; DM2: diabetes mellitus tipo 2; EPOC: enfermedad pulmonar obstructiva crónica; HTA: hipertensión arterial

pacientes. El protocolo de estudio fue aprobado por el comité de ética y se implementó de conformidad con las disposiciones de la Declaración de Helsinki y las directrices de buenas prácticas clínicas.

\section{Resultados}

Durante el tiempo del estudio se realizaron 731 CPRE por patología biliar benigna en tres instituciones, en las que se diagnosticaron $28 \mathrm{PD}$, con una frecuencia del $3.8 \%$ en nuestra población, cuya descripción sociodemográfica se describe en la tabla 1. La edad promedio fue de 41.6 años, siendo en su mayoría de sexo femenino (76.9\%). Las PD fueron clasificadas según lo establecido por Stapfer, et al. ${ }^{6}$ (tabla 2).

En los pacientes en que se sospechó perforación durante la CPRE, el endoscopista realizó la inserción de un stent biliar plástico en 2 (33.3\%) de los pacientes llevados a manejo abierto y en 5 (71.4\%) del grupo de laparoscopia.

A todos los pacientes se les realizó una TC abdominopélvica contrastada, con hallazgo de neumorretroperitoneo y 5 (38.46\%) presentaron neumoperitoneo. Se evidenciaron complicaciones locales pancreáticas, como colección de fluido libre en un $61.5 \%$, colección necrótica aguda en un $30.7 \%$ y colección necrótica 
Tabla 2. Manejo según el tipo de perforación de acuerdo con la clasificación de Stapfer, et al. ${ }^{6}$

\begin{tabular}{|c|c|c|c|}
\hline & Descripción & $\begin{array}{l}\text { Cirugía } \\
\text { abierta } \\
(n=6)\end{array}$ & $\begin{array}{c}\text { Cirugía } \\
\text { laparoscópica } \\
(n=7)\end{array}$ \\
\hline Tipo I & $\begin{array}{l}\text { Lesión de la pared lateral o } \\
\text { medial }\end{array}$ & $3(50 \%)$ & $0(0.0 \%)$ \\
\hline Tipo II & Lesión perivateriana & $1(16.7 \%)$ & $114.3 \%)$ \\
\hline Tipo III & $\begin{array}{l}\text { Lesiones distales del } \\
\text { conducto biliar relacionadas } \\
\text { con instrumentación cerca de } \\
\text { una obstrucción }\end{array}$ & $1(16.7 \%)$ & $3(42.8 \%)$ \\
\hline Tipo IV & Aire libre retroperitoneal & $1(16.7 \%)$ & $3(42.8 \%)$ \\
\hline
\end{tabular}

Tabla 3. Desenlaces quirúrgicos

\begin{tabular}{lcc}
\hline & $\begin{array}{c}\text { Cirugía abierta } \\
(\mathbf{n}=6)\end{array}$ & $\begin{array}{c}\text { Cirugía laparoscópica } \\
(\mathbf{n}=7)\end{array}$ \\
\hline Fístula duodenal & $2(33.3 \%)$ & $0(0 \%)$ \\
Fístula biliar & $3(50 \%)$ & $1(14.2 \%)$ \\
$\begin{array}{l}\text { Estancia hospitalaria } \\
\text { global }\end{array}$ & 33 días & 10.7 días \\
Estancia en cuidados & 14.8 días & 4.2 días \\
intensivos & & \\
Mortalidad & $3(50 \%)$ & $0(0 \%)$ \\
\hline
\end{tabular}

\section{Discusión}

Se han descrito complicaciones secundarias a la CPRE, como pancreatitis $(4,3 \%)$, sangrado $(1,9 \%)$, infecciones y perforaciones del tubo digestivo, de las cuales las PD tienen una incidencia máxima del $1.8 \% \%^{3,8,9}$, como reportan Peñaloza, et al..$^{10}$ en un estudio realizado en nuestra población local, en el que encuentran una incidencia del $1,3 \%$. Sin embargo, en nuestro estudio la incidencia fue del $3.8 \%$, por lo cual creemos que se asoció a la presencia de gastroenterólogos en formación, completando la curva de aprendizaje de la CPRE. No obstante, con mayor frecuencia fueron PD de tipo IV, las cuales presentan una alta tasa de respuesta al manejo médico y una menor mortalidad.

Existen diferentes factores predisponentes que aumentan la probabilidad de una PD, tales como alteraciones anatómicas de la vía biliar (estenosis del conducto biliar y disfunción del esfínter de Oddi) y gastrectomía Billroth II y en Y de Roux. Además, también influyen factores relacionados con la CPRE, la longitud de la esfinterotomía y el manejo del esfinterotomo $0^{5,6,11,12}$, y factores no modificables, como ser mujer joven ${ }^{9}$, tal como se evidencia en nuestro estudio, en el que el $75 \%$ eran mujeres y el $60.7 \%$ eran menores de 55 años.

Stapfer et al. ${ }^{6}$ clasifican las PD en cuatro tipos según su localización. Retuerta, et al..11 describen una incidencia del $80 \%$ de lesiones tipo I y II, pero en nuestro estudio predominaron las lesiones de tipo III y IV, con un total del $82.1 \%$.

La mortalidad en los pacientes de cirugía convencional de nuestro estudio fue del $23.08 \%$, similar a la de otros estudios que reportan un $8-23 \%$, debido a sepsis y falla multiorgánica ${ }^{4,5,12}$. Koc, et al. ${ }^{1}$ encuentran 
una mortalidad para el manejo con cirugía laparoscópica en las PD pos- CPRE de un paciente entre seis manejados con este abordaje, sobre un total de 28 pacientes. En nuestra serie, en el grupo de cirugía mínimamente invasiva la mortalidad fue del $0 \%$. Sin embargo, no hemos encontrado en la literatura estudios con peso epidemiológico que comparen los resultados de la cirugía laparoscópica con los de la cirugía abierta en esta patología.

La sospecha de una PD se basa en la exploración física y los hallazgos paraclínicos e imagenológicos. El paciente puede presentar un dolor abdominal incoercible, signos de irritación peritoneal, fiebre, proteína $C$ reactiva elevada y leucocitosis. Sin embargo, estos hallazgos son muy inespecíficos y deben corroborarse con imágenes diagnósticas ${ }^{6}$.

La radiografía de tórax puede mostrar el neumoperitoneo con una sensibilidad del $50-90 \%$, pero es incapaz en la mayoría de los casos de mostrar con certeza el lugar de la perforación $n^{6,13}$. La ecografía de abdomen puede identificar aire libre intraperitoneal o retroperitoneal, y la formación de abscesos. La TC es el método más sensible para los pacientes con sospecha de perforación intestinal, con una especificidad del $82-90 \%$, ya que permite observar si hay extravasación del medio de contraste, colecciones o neumorretroperitone $0^{5,13,14}$. En nuestro estudio, a todos los pacientes se les realizó una TC abdominal contrastada como método diagnóstico, y el hallazgo más común fue neumorretroperitoneo.

La confirmación del diagnóstico de estas lesiones se puede hacer intraoperatoriamente, mediante su observación directa, o por extravasación del líquido de contraste evidenciada en imágenes posquirúrgicas ${ }^{15}$.

En una revisión sistemática publicada en 2017, con 259 artículos, se encontró que los pacientes llevados a cirugía de forma tardía presentaron un aumento en la complejidad del procedimiento y una mayor morta$\operatorname{lidad}^{2}$. En nuestro estudio, el tiempo promedio de diagnóstico fue de 1.2 días. Por el contrario, los pacientes que fallecieron tuvieron un tiempo de diagnóstico superior a 3 días. Esto concuerda con lo reportado por Cirocchi, et al. ${ }^{2}$, quienes destacan la importancia de un diagnóstico y un manejo tempranos de las PD para reducir las complicaciones asociadas y el riesgo de mortalidad ${ }^{2}$. Así mismo, todos los pacientes tratados satisfactoriamente con manejo médico conservador se diagnosticaron dentro de las primeras 24 horas después de realizada la CPRE.

La baja prevalencia de las PD dificulta un consenso para su manejo'. Se han postulado cuatro tipos de manejo: abierto, laparoscópico, endoscópico y conservador. Sin embargo, esto depende de factores como el tipo de PD, la presencia de líquido libre o aire en la cavidad peritoneal, el estado clínico del paciente, la experiencia del grupo tratante y la disponibilidad de los recursos en el centro hospitalario ${ }^{15-17}$.

La PD de tipo I suele ser una lesión grande, que tiene un manejo inicial quirúrgico. Algunos cirujanos realizan reparación endoscópica, ya que su diagnóstico generalmente es intraoperatorio, dependiendo además de las condiciones del paciente y de la disponibilidad de la tecnología. En nuestro medio, no todas las instituciones cuentan con este tipo de recursos, por lo que no es muy frecuente su uso ${ }^{2,5}$.

Las PD de tipo II pueden tener un manejo médico asociado a stent biliar endoscópico, dependiendo de la estabilidad hemodinámica y del tiempo posterior a la CPRE. Sin embargo, el deterioro clínico del paciente y la no respuesta al manejo médico son indicación de manejo quirúrgico ${ }^{12}$. Un diagnóstico tardío eleva la posibilidad de infección y aumenta la friabilidad del tejido, lo cual dificulta la reparación quirúrgica de la perforación ${ }^{3}$.

Las PD de tipo III se relacionan con la instrumentación usada en la vía biliar y en ellas se recomienda cirugía cuando hay grandes colecciones de fluido retroperitoneal, aunque se pueden resolver de forma espontánea o con drenaje percutáneo y no requerir manejo quirúrgico ${ }^{15}$. En nuestro estudio tuvimos cuatro pacientes con este tipo de PD, de los cuales tres fueron manejados por laparoscopia y uno por cirugía convencional, con una mortalidad del $25 \%$ y complicaciones en el $50 \%$.

Las PD de tipo IV se deben en general al uso de aire comprimido en el tracto gastrointestinal superior. En estas no se realiza manejo quirúrgico, siempre y cuando el paciente no presente signos de irritación peritoneal, dolor abdominal incoercible ni desarrollo de respuesta inflamatoria sistémica persistente ${ }^{15}$. La decisión de llevar a manejo quirúrgico en este grupo de pacientes debe basarse en la evolución individual de cada uno. En nuestro estudio encontramos cuatro pacientes con este tipo de PD, de los cuales tres fueron manejados por laparoscopia y uno por cirugía convencional, con una mortalidad del $0 \%$ y sin complicaciones.

En nuestro estudio, las PD de tipo III y IV fueron manejadas quirúrgicamente, ya que presentaron falla del manejo médico inicial.

Por otro lado, se ha estudiado el uso de stent biliar en las PD como manejo coadyuvante y se ha evidenciado un mejor desenlace en estos pacientes, ya que el stent 
evita la fuga biliar al retroperitoneo, logrando una tasa de rendimiento del tratamiento de hasta un $100 \%$, como muestra la literatura ${ }^{18}$.

En nuestro estudio, en el $53.8 \%$ de los casos se emplearon stents biliares plásticos. El uso de los stents se asoció más al abordaje laparoscópico, en un $71.4 \%$ de los casos. Este hallazgo nos hace pensar que el uso de stent biliar puede estar relacionado con la disminución de la morbilidad en este grupo de pacientes; sin embargo, hay que tener en cuenta que los pacientes manejados por laparoscopia estaban en un mejor estado físico que los llevados a cirugía abierta.

Patil, et al. ${ }^{17}$ realizaron un estudio retrospectivo de un periodo de 12 años, evaluando la morbimortalidad según la técnica quirúrgica. Compararon la reparación primaria más drenaje por laparoscopia frente a la exclusión pilórica y encontraron una menor mortalidad en el grupo de cirugía laparoscópica que en el de cirugía abierta. Así mismo, en nuestro estudio se evidenció una mayor mortalidad en los pacientes con exclusión pilórica más anastomosis gastroyeyunal $(n=3)$. Además, en cuanto a estancia hospitalaria, la media fue mayor en los pacientes con cirugía abierta (33 días) que en aquellos con cirugía laparoscópica (10.7 días). Esto se puede explicar porque los pacientes llevados a cirugía convencional tuvieron un deterioro clínico más grave, determinado por inestabilidad hemodinámica o shock séptico, entre otros, lo cual indica que no todos los pacientes son candidatos a laparoscopia y deben ser adecuadamente seleccionados.

Llama la atención que en la literatura no se reporta la asociación de pancreatitis con PD pos-CPRE; sin embargo, todos nuestros pacientes presentaron esta complicación de origen multifactorial. La European Society of Gastrointestinal Endoscopy postula que los factores de riesgo asociados a la CPRE para desarrollar pancreatitis son ser mujer joven, tener una vía biliar de calibre normal, canulaciones de más de 10 minutos, esfinterotomía o precorte, dilatación del esfínter con balón y extracción de cálculos fallida, entre otros ${ }^{19}$.

A pesar de los resultados, nuestro estudio se ve limitado, al ser retrospectivo, con un sesgo de selección, y por otro lado, por el reducido número de pacientes relacionado con la baja incidencia de la enfermedad, además de la disponibilidad y la experiencia en laparoscopia de los cirujanos participantes en el estudio. No se empleó el abordaje endoscópico para el cierre directo de las PD, ya que en nuestras instituciones no se contaba con este recurso (endoclips).

Aunque la incidencia de PD pos-CPRE tiene un bajo porcentaje, su diagnóstico y manejo temprano reducen de manera importante los desenlaces negativos. Tanto la cirugía abierta como la laparoscopia son herramientas factibles para el manejo de las PD pos-CPRE; no obstante, la elección del procedimiento dependerá del estado clínico del paciente. Un diagnóstico temprano y un adecuado estado clínico permiten realizar una cirugía mínimamente invasiva, mientras que el diagnóstico tardío se asocia a un estado clínico grave y a la elección de cirugía abierta con posible control de daños. Igualmente influyen la comorbilidad, la experiencia del cirujano y la disponibilidad de recursos.

\section{Responsabilidades éticas}

Protección de personas y animales. Los autores declaran que para esta investigación no se han realizado experimentos en seres humanos ni en animales.

Confidencialidad de los datos. Los autores declaran que han seguido los protocolos de su centro de trabajo sobre la publicación de datos de pacientes.

Derecho a la privacidad y consentimiento informado. Los autores declaran que en este artículo no aparecen datos de pacientes.

\section{Financiamiento}

La presente investigación no ha recibido ayudas específicas provenientes de agencias del sector público, del sector comercial ni de entidades sin ánimo de lucro.

\section{Conflictos de intereses}

Los autores declaran no tener conflictos de intereses.

\section{Bibliografía}

1. Koc B, Bircan H, Adas G, Kemik O, Akcakaya A, Yavuz A, et al. Complications following endoscopic retrograde cholangiopancreatography: minimal invasive surgical recommendations. PLoS One. 2014;9:e113073.

2. Cirocchi R, Kelly M, Griffiths E, Tabola R, Sartelli M, Carlini L, et al. A systematic review of the management and outcome of ERCP related duodenal perforations using a standardized classification system. Surgeon. 2017;15:379-87

3. Palanivelu C, Jategaonkar PA, Rangarajan M, Anand NV, Senthilnathan P. Laparoscopic management of a retroperitoneal duodenal perforation following ERCP for periampullary cancer. JSLS. 2008;12:399-402.

4. Temoche-Espinoza E, Rabanal-Galdós $P$, Ruiz-Gutiérrez H. Tratamiento de la perforación duodenal post-CPRE. Revista de la Facultad de Medicina Humana. 2017;17(4). 
5. Armas Ojeda M, Ojeda Marrero V, Roque Castellano C Cabrera Marrero JC, Mathías Gutierrez MP, Ceballos Santos D, et al. Duodenal perforations after endoscopic retrograde cholangiopancreatography. Cir Esp. 2015;93:403-10.

6. Stapfer M, Selby R, Stain S, Katkhouda N, Parekh D, Jabbour N, et al. Management of duodenal perforation after endoscopic retrograde cholangiopancreatography and sphincterotomy. Ann Surg. 2000;232:191-8.

7. Palomeque Jiménez A, González Puga C, Pérez Cabrera $B$, Jiménez Ríos JA. Tratamiento conservador de la perforación duodenal tras realización de colangiopancreatografía retrógrada endoscópica terapéutica. Gastroenterol Hepatol. 2015;38:285-6.

8. Rustagi T, Jamidar P. Endoscopic retrograde cholangiopancreatography-related adverse events. Gastrointest Endosc Clin North Am. 2015;25:97-106.

9. Machado NO. Management of duodenal perforation post-endoscopic retrograde cholangiopancreatography. When and whom to operate and what factors determine the outcome? A review article. JOP. 2012;13:18-25.

10. Peñaloza-Ramírez $A$, Leal-Buitrago $C$, Rodríguez-Hernández $A$. Adverse events of ERCP at San José Hospital of Bogotá (Colombia). Rev Esp Enferm Dig. 2009;101:837-49.

11. Mateo Retuerta J, Chaveli Díaz C, Goikoetxea Urdiain A, Sainz Villacampa B, Sara Ongay MJ, Ínigo Noain JJ. Perforaciones post colangiopancreatografía endoscópica retrógrada (CPRE). Manejo quirúrgico. An Sist Sanit Navar. 2017;40:145-51.
12. Kwon $\mathrm{Cl}$, Song $\mathrm{SH}$, Hahm KB, Ko KH. Unusual complications related to endoscopic retrograde cholangiopancreatography and its endoscopic treatment. Clin Endosc. 2013;46:251-9.

13. Shin D, Rahimi H, Haroon S, Merritt A, Vemula A, Noronha A, et al. Imaging of gastrointestinal tract perforation. Radiol Clin North Am. 2020;58:19-44.

14. Bostancı Ö, Battal M, Yazıcı P, Demir U, Alkım C. Management of iatrogenic injuries due to endoscopic sphincterotomy: surgical or conservative approaches. Turk J Surg. 2018;34:24-7.

15. Kumbhari V, Sinha A, Reddy A, Afghani E, Cotsalas D, Patel YA, et al. Algorithm for the management of ERCP-related perforations. Gastrointest Endosc. 2016;83:934-43.

16. Lee $\mathrm{TH}, \mathrm{Han} \mathrm{JH}$, Park SH. Endoscopic treatments of endoscopic retrograde cholangiopancreatography-related duodenal perforations. Clin Endosc. 2013;46:522-8.

17. Patil NS, Solanki N, Mishra PK, Sharma BC, Saluja SS. ERCP-related perforation: an analysis of operative outcomes in a large series over 12 years. Surg Endosc. 2020;34:77-87.

18. Canena J, Liberato M, Horta D, Romão C, Coutinho A. Short-term stenting using fully covered self-expandable metal stents for treatment of refractory biliary leaks, post sphincterotomy bleeding, and perforations. Surg Endosc. 2013;27:313-24.

19. Dumonceau JM, Andriulli A, Elmunzer BJ, Mariani A, Meister T, Deviere J, et al Prophylaxis of post-ERCP pancreatitis: European Society of Gastrointestinal Endoscopy (ESGE) Guideline - updated June 2014. Endoscopy. 2014;46:799-81. 